\title{
Transdermal buprenorphine-interesting observations on the metabolite norbuprenorphine levels in elderly subjects
}

\author{
Nuggehally R. Srinivas
}

Received: 7 February 2013 /Accepted: 18 March 2013 /Published online: 16 April 2013

(C) Springer-Verlag Berlin Heidelberg 2013

Dear Sir,

Buprenorphine is a narcotic analgesic which is typically used in patients with opioid dependence [1]. The use of low potency opioid analgesics as a second-line treatment may have advantages in elderly osteoarthritis patients [2,3]. In this context, the ready availability of transdermal patches of buprenorphine would make it an attractive treatment option, especially in elderly patients.

The recently published work of Al-Tawil et al. [4] has explored the pharmacokinetics (PK) of buprenorphine and its metabolite norbuprenorphine in two groups of individuals: elderly ( $\geq 75$ years) and young ( $50-60$ years). The agerelated influence on the elimination pathway of drugs provided the basis for this interesting comparative PK/safety study. The sample size chosen in this study had $80 \%$ power to obtain the $90 \%$ confidence interval of the $\mathrm{AUC}_{\text {tau }}$ ratio (elderly:young) to be contained within $80-125 \%$. Due to higher variability in the data sets, bioequivalence was not concluded between the two groups; however, similarity in the point estimate of buprenorphine exposure led to the recommendation that dosage adjustment was not necessary in the use of buprenorphine transdermal patches in the elderly subjects [4].

My intention in this letter is to further evaluate the data and provide additional perspectives, taking into consideration both the parent and metabolite data which were generated in this study [4]. For the purpose of this

N. R. Srinivas $(\triangle)$

Integrated Drug Development, Suramus Biopharm, J.P. Nagar, I

Phase,

Bangalore, India 560078

e-mail: srini.suramus@yahoo.com assessment, the mean values and the maximum values reported for $\mathrm{AUC}_{\text {tau }}, \mathrm{C}_{\text {maxss }}$ and $\mathrm{C}_{\text {minss }}$ of buprenorphine and norbuprenorphine were utilized [4]. In total, there were six paired sets of parent and metabolite data in the elderly and young subjects. The data were evaluated in two steps. In step 1 , for each $\mathrm{PK}$ parameter $\left(\mathrm{AUC}_{\text {tau }}\right.$, $\mathrm{C}_{\text {maxss }}, \mathrm{C}_{\text {minss }}$ ), the norbuprenorphine/buprenorphine ratio was computed separately for the young and elderly groups; in step 2, the relative change observed for elderly was computed as a quotient of elderly/young metabolic ratios.

As evidenced in Table 1, examination of the relative change for the various parameters appears to suggest that there was a somewhat higher presence of metabolite in the elderly group. This finding was quite pronounced in the maximum values reported for $\mathrm{AUC}_{\mathrm{tau}}, \mathrm{C}_{\text {maxss }}$ and $\mathrm{C}_{\text {minss }}$ in the study [4].

Due to the availability of a large data set of buprenorphine and norbuprenorphine in this study [4], it was possible to tease out a qualitative evidence of a higher circulatory metabolite levels in the elderly relative to young subjects. From a speculative reasoning, this interesting observation would signify either norbuprenorphine formation was higher in the elderly or the clearance/elimination of norbuprenorphine was impaired in the elderly, leading to its accumulation.

Intuitively, the proposition of the higher formation of norbuprenorphine appears to be flawed because it would mean relatively more enzymatic activity in the elderly versus young, which is contrary to the known fact that enzymatic clearance or metabolism in general decreases with age [5]. On the contrary, the maximum $\mathrm{C}_{\text {minss }}$ metabolite/parent 
Table 1 Assessment of norbuprenorphine/ buprenorphine ratios - computation of relative change in elderly versus young
Pharmacokinetic parameters:

$\mathrm{AUC}_{\text {tau }}$, Area under the curve at end of first dose interval; $\mathrm{C}_{\text {maxss }}$, peak concentration at steady state; $\mathrm{C}_{\text {minss }}$, trough concentration at steady state

${ }^{\mathrm{a}} \mathrm{E} / \mathrm{Y}$, Elderly/young

\begin{tabular}{|c|c|c|c|c|}
\hline Analyte & Young & Elderly & Young & Elderly \\
\hline & \multicolumn{2}{|c|}{ Mean $\mathrm{AUC}_{\mathrm{tau}}(\mathrm{pg} / \mathrm{h} / \mathrm{ml})$} & \multicolumn{2}{|c|}{ Maximum $\mathrm{AUC}_{\mathrm{tau}}(\mathrm{pg} / \mathrm{h} / \mathrm{ml})$} \\
\hline Buprenorphine (B) & $11,309.33$ & 9939.87 & 22464 & 19842 \\
\hline Norbuprenorphine (NB) & 2922 & 3063.23 & 6631.2 & 7669.2 \\
\hline Ratio (B/NB) & 0.258 & 0.308 & 0.295 & 0.386 \\
\hline \multirow[t]{2}{*}{ Relative change $(\mathrm{E} / \mathrm{Y})^{\mathrm{a}}$} & $19 \%$ & & $31 \%$ & \\
\hline & \multicolumn{2}{|c|}{ Mean $\mathrm{C}_{\operatorname{maxss}}(\mathrm{pg} / \mathrm{ml})$} & \multicolumn{2}{|c|}{ Maximum $\mathrm{C}_{\operatorname{maxss}}(\mathrm{pg} / \mathrm{ml})$} \\
\hline Buprenorphine (B) & 83.46 & 72.15 & 169 & 153 \\
\hline Norbuprenorphine (NB) & 21.73 & 20.46 & 50 & 56.5 \\
\hline Ratio (B/NB) & 0.260 & 0.284 & 0.296 & 0.369 \\
\hline \multirow[t]{2}{*}{ Relative change (E/Y) } & $9 \%$ & & $25 \%$ & \\
\hline & \multicolumn{2}{|c|}{ Mean $\mathrm{C}_{\text {minss }}(\mathrm{pg} / \mathrm{ml})$} & \multicolumn{2}{|c|}{ Maximum $\mathrm{C}_{\text {minss }}(\mathrm{pg} / \mathrm{ml})$} \\
\hline Buprenorphine (B) & 47.71 & 47.45 & 110 & 93.9 \\
\hline Norbuprenorphine (NB) & 13.85 & 16.33 & 31.7 & 42.7 \\
\hline Ratio (B/NB) & 0.290 & 0.344 & 0.288 & 0.455 \\
\hline Relative change (E/Y) & $18 \%$ & & $58 \%$ & \\
\hline
\end{tabular}

ratio was $58 \%$ (Table 1), suggesting that slow clearance of norbuprenorphine may reasonably explain the observed finding since trough levels are likely to be governed by clearance mechanisms. Since norbuprenorphine clearance is governed by the saturable glucuronidation pathway by UGT1A3 only (as opposed to UGT1A1, UGT1A3 and UGT2B7 in buprenorphine) [6, 7], one may suspect that there could have been a natural slowing down of such a pathway in the elderly. Interestingly, corroborative evidence has been reported in a recent study, where buprenorphine has been shown to inhibit the glucuronidation of norbuprenorphine, the only clearance pathway for norbuprenorphine, in multiple in vitro systems [8]. The results of this recent study assumes greater importance since 3-norbuprenorphine glucuronide was found to be a major circulatory species with five- to 13 -fold higher levels as compared to 3-buprenorphine glucuronide in several clinical studies following buprenorphine dosing $[9,10]$.

Interestingly, although the formation of nurbuprenorphine suggests a clear route dependency, as evidenced by the norbuprenorphine/buprenorphine exposure ratios following transdermal (average 0.32), sublingual (average 1.52) and intravenous (average 0.19) drug administration of buprenorphine $[4,9,11]$, the involvement of the glucuronidation pathway for the clearance of norbuprenorphine is highly inevitable regardless of the route of administration. Hence, the qualitative evidence gathered by the cursory data analysis in this report needs further probing because increased circulatory levels of norbuprenorphine in the elderly may contribute to several safety issues, including respiratory depression.

\section{References}

1. Johnson RE, Chutuape MA, Strain EC, Walsh SL, Stitzer ML, Bigelow GE (2000) A comparison of levomethadol acetate, buprenorphine and methadone for opioid dependence. N Engl J Med 343:1290-1297

2. Budd K (2003) Buprenorphine and the transdermal system: the ideal match in pain management. Int J Clin Pract Suppl (133):9-14

3. Swedish Medical Products Agency (2004) Treatment of osteoarthritis [in Swedish]. Swedish Medical Products Agency 13:19-25

4. Al-Tawil N, Odar-Cederlöf I, Berggren AC, Johnson HE, Persson J (2013) Pharmacokinetics of ransdermal buprenorphine patch in the elderly. Eur J Clin Pharmacol 69(2):143-149

5. Klotz U (2009) Pharmacokinetics and drug metabolism in the elderly. Drug Metab Rev 41(2):67-76

6. Chang Y, Moody DE (2009) Glucuronidation of buprenorphine and norbuprenorphine by human liver microsomes and UDPglucuronosyltransferases. Drug Metab Lett 3(2):101-107

7. Rouguieg K, Picard N, Sauvage FL, Gaulier JM, Marquet P (2010) Contribution of the different UDP-glucuronosyltransferase (UGT) isoforms to buprenorphine and norbuprenorphine metabolism and relationship with the main UGT polymorphisms in a bank of human liver microsomes. Drug Metab Dispos 38(1):40-45

8. Oechsler S, Skopp G (2010) An in vitro approach to estimate putative inhibition of buprenorphine and norbuprenorphine glucuronidation. Int J Legal Med 124(3):187-194

9. Moody DE, Fang WB, Morrison J, McCance-Katz E (2011) Gender differences in pharmacokinetics of maintenance dosed buprenorphine. Drug Alcohol Depend 118(2-3):479-483

10. Concheiro M, Jones HE, Johnson RE, Choo R, Huestis MA (2011) Preliminary buprenorphine sublingual tablet pharmacokinetic data in plasma, oral fluid, and sweat during treatment of opioiddependent pregnant women. Ther Drug Monit 33(5):619-626

11. Huestis MA, Cone EJ, Pirnay SO, Umbricht A, Preston KL (2012) Intravenous buprenorphine and norbuprenorphine pharmacokinetics in humans. Drug Alcohol Depend. doi: 10.1016/j.drugalcdep. 2012.11.014 